do not know Dr. Maclean, the suspicion that the whole of the cases in THE LANCET for 7 th January, were a tissue of falsehoods, concocted in the region of Russell-square, London?

It is a law of evidence, that when extraordinary events, such as were never before known to happen, are asserted to have taken place, they must be proven by the testimony of more than one competent witness. I would ask, then, what medical man of education in Thurso saw the cure of the closed pupil, and of the staphylomatous eye, accomplished in this case by Dr. Maclean, with the aid of prussic acid?

8. In Dr. Maclean's fifth case, the motions of the iris were natural, but from over-exertion of the eyes there was pain in the orbits, intolerance of light, and some obscurity of vision. These symptoms subsided under rest and the use of the acid. May not as much of the benefit be attributable to the former of these means of cure as to the latter?

9. In Dr. Maclean's sixth case, a woman is affected with capsular cataract for three or four years, prussic acid vapour is used, and the "diseased structure" is absorbed. Does the doctor mean that the opake capsule was absorbed? Might it not have been merely a deposition of lymph on the surface of the capsule, stimulated to absorption by the acid vapour? Was this case seen by any other medical man, and recognised as one of true cataract, having its seat in the capsule?

10. The seventh case is merely one of chronic ophthalmia, which subsides under the use of the acid, and might, possibly, have done so under the influence of the vapour of turpentine, ammonia, laudanum, or even of mere warm water, had any of these been tried.

$I$ have written these remarks in no unfriendly spirit towards Dr. Maclean, but from a sincere desire to elicit the truth. I trust the doctor will be so good as to give an answer to the queries $I$ have taken the liberty of putting. In the meantime, as the author of certain queries formerly published in THE LANCET, regarding the effects of prussic acid on the organs of vision, I beg leave to thank Dr. Maclean for his commanication, and am, \&c.

Aberdeen, Jan. 11, 1843.

Borvssus.

New Charpie. - The French Acad. des Sciences was lately presented with a specimen of charpie manufactured by a new process, and which it is supposed will possess an advantage over that now in use, by its greater purity. The thread is successively submitted to the action of acid, chlorine, alkaline, and caustic alkaline solutions, before being washed in pure water, and dried, beaten, cut, and carded.

\section{USE OF THE NITRATE OF SILVER}

IN THE

SUBDUCTION OF ERYTHEMA.

\section{To the Editor of The Lancet.}

SiR,-A healthy boy met with an accident by which the right cheek was lacerated from the inner canthus of the eye to below the angle of the mouth. The parts were brought together by adhesive straps, but contact could not be very well preserved immediately subjacent to the eye itself. This, however, was effected, and union facilitated, by placing underneath the plaster a small piece of moistened card-paper. All went on well for about a week, when the lower lid, on the under edge of which the card-paper necessarily pressed, became erythematic. The paper was removed, but still the swelling remained. The lid was moistened with water, and a stick of nitrate of silver was passed freely over every part of it without producing any pain, by which the swelling. was transferred from the lower to the upper lid. The nitrate of silver was again used, which caused considerable pain, and the inflammation was subdued. My little patient was living with his parents, several miles from my residence, and it was on visiting him that I found, rather unexpectedly, that the inflammation was unsubdned, notwithstanding the card-paper had been removed. Leeches might have been advantageously applied in ordinary cases, but in this instance they would have interfered with my treatment of the wound, and, besides, they could not have been procured without considerable loss of time. The tincture of iodine, also, I have no doubt, would have acted specifically, but I had none with me, and as there was the greatest necessity for prompt treatment, I had recourse to the lunar caustic, which I had occasion to apply to the wound. A few days after this $I$ met with Dr. Hocken's excellent paper, describing a new plan of treating strumous conjunctivitis (page 382), which he appears to have been taught by Mr. Wormald, at St. Bartholomew's Hospital, but the latter gentleman repudiates the idea of its being new. Probably the application of the nitrate of silver in the treatment of local inflammation in general, originated with Mr. Higginbottom. At all events I believe that his excellent treatise on the subject has contributed towards its being more extensively used. Dr. Hocken's paper, however, affords a striking illustration of the importance of the remedy, for the publication of which I feel much indebted to that gentleman. I am, Sir, your obedient servant,

$$
\text { Richard Lanyon, M.D., F.A.S. }
$$

Lostwithiel, Jan. 9, 1843. 